\title{
Understanding caustic crossings in giant arcs: Characteristic scales, event rates, and constraints on compact dark matter
}

\author{
Masamune Oguri, ${ }^{1,2,3}$ Jose M. Diego, ${ }^{4}$ Nick Kaiser, ${ }^{5}$ Patrick L. Kelly, ${ }^{6}$ and Tom Broadhurst ${ }^{7,8}$ \\ ${ }^{1}$ Research Center for the Early Universe, University of Tokyo, Tokyo 113-0033, Japan \\ ${ }^{2}$ Department of Physics, University of Tokyo, Tokyo 113-0033, Japan \\ ${ }^{3}$ Kavli Institute for the Physics and Mathematics of the Universe (Kavli IPMU, WPI), \\ The University of Tokyo, Chiba 277-8582, Japan \\ ${ }^{4}$ IFCA, Instituto de Física de Cantabria (UC-CSIC), Avenida de Los Castros s/n, 39005 Santander, Spain \\ ${ }^{5}$ Institute for Astronomy, University of Hawaii, 2680 Woodlawn Drive, Honolulu, \\ Hawaii 96822-1839, USA \\ ${ }^{6}$ School of Physics and Astronomy, University of Minnesota, 116 Church Street SE, \\ Minneapolis, Minnesota 55455, USA \\ ${ }^{7}$ Department of Theoretical Physics, University of the Basque Country, Bilbao 48080, Spain \\ ${ }^{8}$ IKERBASQUE, Basque Foundation for Science, Alameda Urquijo, 36-5 48008 Bilbao, Spain
}

(Received 5 October 2017; published 19 January 2018)

\begin{abstract}
The recent discovery of fast transient events near critical curves of massive galaxy clusters, which are interpreted as highly magnified individual stars in giant arcs due to caustic crossing, opens up the possibility of using such microlensing events to constrain a range of dark matter models such as primordial black holes and scalar field dark matter. Based on a simple analytic model, we study lensing properties of a point mass lens embedded in a high magnification region, and we derive the dependence of the peak brightness, microlensing time scales, and event rates on the mass of the point mass lens, as well as the radius of a source star that is magnified. We find that the lens mass and source radius of the first event MACS J1149 Lensed Star 1 (LS1) are constrained, with the lens mass range of $0.1 M_{\odot} \lesssim M \lesssim 4 \times$ $10^{3} M_{\odot}$ and the source radius range of $40 R_{\odot} \lesssim R \lesssim 260 R_{\odot}$. In the most plausible case with $M \approx 0.3 M_{\odot}$ and $R \approx 180 R_{\odot}$, the source star should have been magnified by a factor of $\approx 4300$ at the peak. The derived lens properties are fully consistent with the interpretation that MACS J1149 LS1 is a microlensing event produced by a star that contributes to the intracluster light. We argue that compact dark matter models with high fractional mass densities for the mass range $10^{-5} M_{\odot} \lesssim M \lesssim 10^{2} M_{\odot}$ are inconsistent with the observation of MACS J1149 LS1 because such models predict too low magnifications. Our work demonstrates a potential use of caustic crossing events in giant arcs to constrain compact dark matter.
\end{abstract}

DOI: 10.1103/PhysRevD.97.023518

\section{INTRODUCTION}

Recently, Kelly et al. [1] reported the discovery of MACS J1149 Lensed Star 1 (LS1, also known as "Icarus"), a faint transient near the critical curve of the massive cluster MACS J1149.6+2223. The transient is interpreted as a luminous star in the host galaxy of supernova Refsdal [2] at $z=1.49$, which is magnified by a compact object very close to the critical curve of the foreground lens. The light curve is consistent with caustic crossing of the background star, and from the comparison with ray-tracing simulations it was suggested that the star was probably magnified by a factor of several thousands at the peak brightness. There was also an additional transient ("Iapyx") detected at roughly the same distance from the critical curve of the cluster but on the opposite side, which can be the counterimage of LS1. Furthermore, Rodney et al. [3] reported two peculiar fast transients ("Spock") behind the cluster MACS J0416.1-2403 in a strongly lensed galaxy at $z=1.0054$. While the Spock events can be explained by the outburst of a luminous blue variable star or a recurrent nova, one possible interpretation is that these two events are also caustic crossing events.

These caustic crossing events in giant arcs behind massive clusters remarkably differ from traditional microlensing observations. Microlensing observations in our Galaxy or in nearby galaxies (e.g., [4-11]) are usually produced by isolated stars (or compact objects), whereas caustic crossing events in giant arcs are produced by stars embedded in high magnification regions due to the cluster potential. As shown in previous works (e.g., [12-16]), microlensing properties are significantly modified by the presence of such convergence and shear field due to the cluster potential.

Perhaps quasar microlensing (e.g., [17-24]) more resembles caustic crossing events in giant arcs in the sense that it is also caused by stars embedded in high magnification 
regions due to the lensing galaxy. However there are several notable differences between microlensing in giant arcs and quasar microlensing. For example, in the former case the source is a star, whereas in the latter case the source is a quasar. While the internal structure of a quasar is complicated with largely different sizes for different wavelengths, the surface brightness distribution of a star is uniform (neglecting limb darkening) and its radius can be predicted from observations of nearby stars. Also, the radius of a star is typically much smaller than the size of the light-emitting region of a quasar. The smaller radius translates into a higher maximum magnification that scales as the inverse of the square root of the radius (see Sec. II). In addition, while typical magnifications of the brightest images of lensed quasars are $\approx 10-20$, MACS J1149 LS1 is observed very close to the critical curve of the macro lens model where the magnification due to the macro lens model is estimated to be $\gtrsim 300$. Such high magnification of the macro mass model is possible because the giant arc directly crosses the critical curve and many stars in the giant arcs are located very near the critical curve.

Caustic crossing events in giant arcs are new phenomena that may probe a different parameter space from previously known microlensing events. This possibility of observing highly magnified images of individual stars in giant arcs was first discussed in [25], although only the smooth cluster potential was considered in that work. Motivated by the discovery of MACS J1149 LS1, the authors of [26,27] revisited this problem. They argued that even a small fraction of compact objects in the lens disrupts the critical curve into a network of microcaustics and drastically modifies the microlensing properties near the critical curve. Even if dark matter consists entirely of a smooth component, such compact objects are expected to exist, like for instance the stars that are responsible for the so-called intracluster light (ICL). Given the drastic change of lensing properties near the critical curve, it has been argued that caustic crossing events in giant arcs may serve as a powerful probe of a range of dark matter scenarios such as primordial black holes (PBHs) [28-32] and scalar field dark matter [33-35].

In this paper, we adopt a simple analytic model that consists of a point mass lens and a constant convergence and shear component, and we study basic microlensing properties of this lens model. The result is used to derive characteristic scales of caustic crossing events in giant arcs and their dependences on the lens and source properties. The result is used to interpret MACS J1149 LS1 and place constraints on the lens mass as well as the property of the source star. We also discuss the event rates of caustic crossing. Such analytic studies of caustic crossing events should complement the ray-tracing simulations presented in $[1,26]$ for which repeating simulations with many different model parameters may be computationally expensive. Our approach more resembles that in [27], which appeared recently.
This paper is organized as follows. We present an analytic lens model which sets the theoretical background in Sec. II. We then discuss what kinds of constraints we can place from observed caustic crossing events in Sec. III. We apply our results to MACS J1149 LS1 to derive constraints on lens and source properties of this particular microlensing event in Sec. IV. We discuss event rates and derive expected event rates for MACS J1149 LS1 as well as for general cases in Sec. V. In Sec. VI, we discuss constraints on compact dark matter in the presence of ICL. Finally we summarize our results in Sec. VII. Throughout the paper we adopt a cosmological model with the matter density $\Omega_{\mathrm{m}}=0.3$, cosmological constant $\Omega_{\Lambda}=0.7$, and the Hubble constant $H_{0}=70 \mathrm{~km} \mathrm{~s}^{-1} \mathrm{Mpc}^{-1}$.

\section{GENERAL THEORY}

Here we summarize basic properties of gravitational lensing by a point mass lens embedded in a high magnification region. The high magnification region concentrates around the caustics that are produced by a macro lens model of, e.g., a massive cluster of galaxies, and we consider a perturbation by a compact object to a highly magnified background object (star) near the caustics. The lensing properties of such a compound system have been studied in depth in the literature [12-16] and more recently by $[26,27]$ in the context of interpreting MACS J1149 LS1 [1]. We present some key results which are necessary for the discussions in the following sections.

\section{A. Lens equation}

We consider a point mass lens in a constant convergence $(\bar{\kappa})$ and shear $(\bar{\gamma})$ field which comes from a macro lens model. Then we have

$$
\begin{aligned}
& \mu_{\mathrm{t}}^{-1}=1-\bar{\kappa}-\bar{\gamma}, \\
& \mu_{\mathrm{r}}^{-1}=1-\bar{\kappa}+\bar{\gamma} .
\end{aligned}
$$

The total magnification by the macro lens model is $\bar{\mu}=\mu_{\mathrm{t}} \mu_{\mathrm{r}}$. We consider a region near the tangential critical curve where $\mu_{\mathrm{t}}^{-1} \approx 0$ gives rise to the high magnification.

A point mass lens with mass $M$, in the absence of the macro mass model, has the Einstein radius of

$$
\theta_{\mathrm{E}}=\left(\frac{4 G M}{c^{2}} \frac{D_{l s}}{D_{o s} D_{o l}}\right)^{1 / 2} .
$$

The lens equation for the point mass lens embedded in the macro lens model is

$$
\begin{aligned}
& \beta_{1}=\frac{\theta_{1}}{\mu_{\mathrm{r}}}-\frac{\theta_{\mathrm{E}}^{2} \theta_{1}}{\theta^{2}}, \\
& \beta_{2}=\frac{\theta_{2}}{\mu_{\mathrm{t}}}-\frac{\theta_{\mathrm{E}}^{2} \theta_{2}}{\theta^{2}} .
\end{aligned}
$$


Note that $\left(\beta_{1}, \beta_{2}\right)$ is the position of the source and $\left(\theta_{1}, \theta_{2}\right)$ is the position of the image. The origin of the coordinates is taken at the position of the point mass lens. For simplicity we assume that the shear direction is aligned with the $x$ axis. The inverse magnification matrix is

$\frac{\partial \vec{\beta}}{\partial \vec{\theta}}=\left(\begin{array}{cc}\mu_{\mathrm{r}}^{-1}+\theta_{\mathrm{E}}^{2} \cos (2 \phi) / \theta^{2} & -\theta_{\mathrm{E}}^{2} \sin (2 \phi) / \theta^{2} \\ -\theta_{\mathrm{E}}^{2} \sin (2 \phi) / \theta^{2} & \mu_{\mathrm{t}}^{-1}-\theta_{\mathrm{E}}^{2} \cos (2 \phi) / \theta^{2}\end{array}\right)$,

where $\phi$ is the polar angle of $\left(\theta_{1}, \theta_{2}\right)$. The magnification is

$$
\mu^{-1}=\left(\mu_{\mathrm{t}} \mu_{\mathrm{r}}\right)^{-1}-\left(\mu_{\mathrm{r}}^{-1}-\mu_{\mathrm{t}}^{-1}\right) \cos (2 \phi) \frac{\theta_{\mathrm{E}}^{2}}{\theta^{2}}-\frac{\theta_{\mathrm{E}}^{4}}{\theta^{4}} .
$$

\section{B. Critical curve and caustic}

The critical curve can be derived from $\mu^{-1}=0$. Specifically,

$$
\begin{aligned}
\left(\frac{\theta}{\theta_{\mathrm{E}}}\right)^{2}= & \frac{\cos (2 \phi)}{2}\left(\mu_{\mathrm{t}}-\mu_{\mathrm{r}}\right) \\
& \times\left[1 \pm \sqrt{1+\frac{4 \mu_{\mathrm{t}} \mu_{\mathrm{r}}}{\left(\mu_{\mathrm{t}}-\mu_{\mathrm{r}}\right)^{2} \cos ^{2}(2 \phi)}}\right] \\
\approx & \frac{\mu_{\mathrm{t}} \cos (2 \phi)}{2}\left[1 \pm \sqrt{1+\frac{4 \mu_{\mathrm{r}}\left(\operatorname{sgn} \mu_{\mathrm{t}}\right)}{\left|\mu_{\mathrm{t}}\right| \cos ^{2}(2 \phi)}}\right] .
\end{aligned}
$$

A similar equation is also given in [26]. The caustic is obtained by converting the critical curve in the image plane to the source plane via the lens equation. Figure 1 shows the critical curves and caustics for positive and negative parities, which have already been given in the literature (e.g., [12]).

The solutions of Eq. (8) depend on the sign of $\mu_{\mathrm{t}}$ (or parity), which is different on each side of the critical curve. $\mu_{\mathrm{r}}$ maintains the same sign on both sides of the critical curve, and without loss of generality, we assume that $\mu_{\mathrm{r}}$ is always positive (i.e., tangential critical curves).

\section{Positive parity case: $\mu_{\mathrm{t}}>0$}

From Eq. (8) we can easily estimate the size of the critical curve along the $\theta_{1}$ and $\theta_{2}$ axes. Along $\theta_{1}$ [or horizontal axis, i.e., $\cos (2 \phi)=1$ ] we have

$$
\theta_{\text {crit }, 1} \approx \sqrt{\mu_{\mathrm{t}}} \theta_{\mathrm{E}}
$$

and along $\theta_{2}$ [or vertical axis, i.e., $\cos (2 \phi)=-1$ ] we have

$$
\theta_{\text {crit }, 2} \approx \sqrt{\mu_{\mathrm{r}}} \theta_{\mathrm{E}}
$$

Using the lens equation, the corresponding size of the caustic along the $\beta_{1}$ and $\beta_{2}$ axes is estimated as
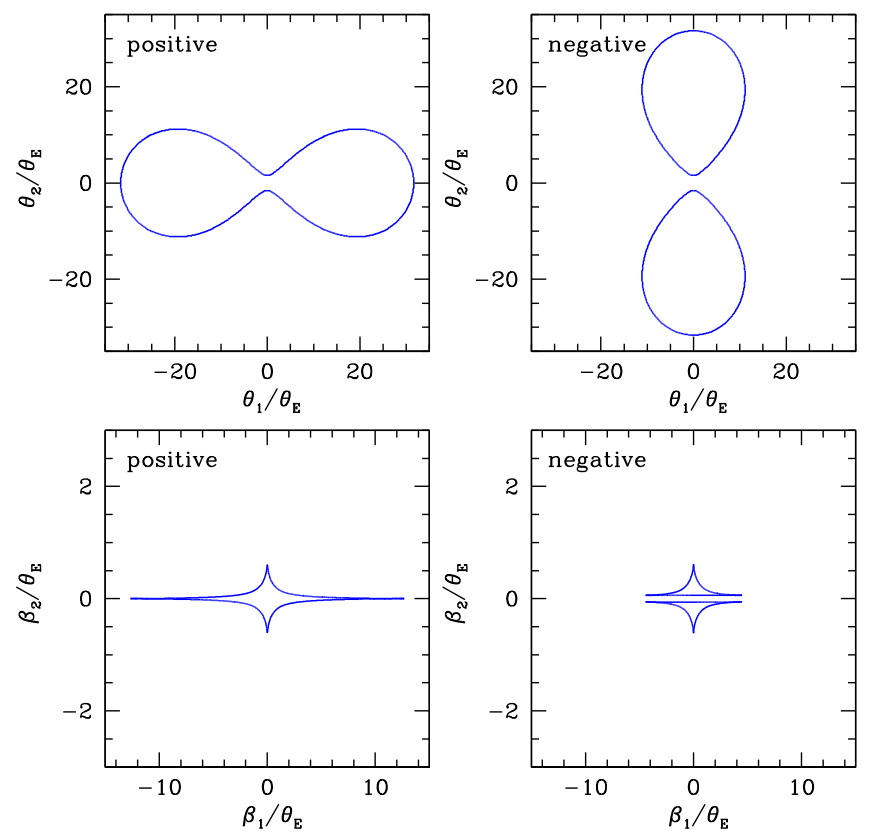

FIG. 1. Critical curves (upper panels) and caustics (lower panels) of a point mass lens in the high magnification region. Left: Positive parity case with $\mu_{\mathrm{t}}^{-1}=0.001$ and $\mu_{\mathrm{r}}^{-1}=0.401$. Right: Negative parity case with $\mu_{\mathrm{t}}^{-1}=-0.001$ and $\mu_{\mathrm{r}}^{-1}=0.399$. These are computed with GLAFIC [36]. Note the difference of scales between the $x$ and $y$ axes in the lower panels.

$$
\begin{aligned}
& \beta_{\text {caus }, 1} \approx \frac{\sqrt{\mu_{\mathrm{t}}}}{\mu_{\mathrm{r}}} \theta_{\mathrm{E}}, \\
& \beta_{\text {caus }, 2} \approx \frac{1}{\sqrt{\mu_{\mathrm{r}}}} \theta_{\mathrm{E}} .
\end{aligned}
$$

We also want to know the typical width of the caustic. As shown in Fig. 1, it becomes very long and thin. Given that the size of the critical curves at the region of interest is $\theta_{\text {crit }} \approx \mathcal{O}\left(\sqrt{\mu_{\mathrm{t}}} \theta_{\mathrm{E}}\right)$, from Eq. (5) we can infer the typical width of the caustic to be (assuming $\sqrt{\mu_{\mathrm{t}}} \gg 1$ )

$$
\beta_{\mathrm{w}} \approx \frac{\theta_{\mathrm{E}}}{\sqrt{\mu_{\mathrm{t}}}}
$$

which implies that the total area enclosed by the caustic is not significantly enhanced compared with the case of an isolated point mass lens. Figure 1 indicates that the area enclosed by the caustic at around $\beta_{1} \approx 0$ has the same order.

\section{Negative parity case: $\mu_{\mathrm{t}}<0$}

In this case, the critical curves do not form along the $\theta_{1}$ axis because the right-hand side of Eq. (8) is always negative. On the other hand, there are two solutions of Eq. (8) along the $\theta_{2}$ axis, which we denote $\theta_{\text {crit, }+}$ and $\theta_{\text {crit,-- }}$. They are 


$$
\begin{gathered}
\theta_{\text {crit, }+} \approx \sqrt{\left|\mu_{\mathrm{t}}\right|} \theta_{\mathrm{E}}, \\
\theta_{\text {crit, }-} \approx \sqrt{\mu_{\mathrm{r}}} \theta_{\mathrm{E}} .
\end{gathered}
$$

The corresponding size of the caustic along the $\beta_{2}$ axis is

$$
\begin{aligned}
& \beta_{\text {crit, }+} \approx \frac{2}{\sqrt{\left|\mu_{\mathrm{t}}\right|}} \theta_{\mathrm{E}} \\
& \beta_{\text {crit, }-} \approx \frac{1}{\sqrt{\mu_{\mathrm{r}}}} \theta_{\mathrm{E}} .
\end{aligned}
$$

We also want to estimate the size of the critical curve and caustic along the $\theta_{1}$ direction. First, the maximum $\theta_{1}$ of the critical curves is estimated as follows. In the region of interest, we can approximate $\left(\theta / \theta_{\mathrm{E}}\right)^{2} \approx \mu_{\mathrm{t}} \cos (2 \phi)$. At the maximum along $\theta_{1}, \theta_{\text {crit,max }}, \theta_{1}^{2}=\theta^{2} \cos ^{2} \phi$ must be stationary, leading to $\cos \phi=1 / 2$ and $\cos (2 \phi)=-1 / 2$. Therefore,

$$
\theta_{\text {crit,max }} \approx \sqrt{\frac{\left|\mu_{\mathrm{t}}\right|}{8}} \theta_{\mathrm{E}}
$$

The corresponding size of the caustic along the $\beta_{1}$ direction is

$$
\beta_{\text {caus,max }} \approx \frac{1}{\mu_{\mathrm{r}}} \sqrt{\frac{\left|\mu_{\mathrm{t}}\right|}{8}} \theta_{\mathrm{E}}
$$

We can use the same argument as above to show that the typical width of the caustics is given by Eq. (13). Thus, the area enclosed by the caustics is again largely unchanged compared with the case of an isolated point mass lens, which can also be seen in Fig. 1. By comparing Eqs. (11) and (19), it is found that the length of the caustic is longer in the positive parity than in the negative parity by a factor of $2 \sqrt{2}$. However, Fig. 1 indicates that in the negative parity there are twice as many caustic crossings for each lens, which compensates for the shorter length of the caustic when we estimate the rate of caustic crossings (see Sec. V).

The source plane region surrounded by $\beta= \pm \beta_{\text {crit, }+}$ demagnifies the star. As an example, we consider multiple images for a source placed at the origin $\left(\beta_{1}, \beta_{2}\right)=(0,0)$. From the lens equation, we find that there are two multiple images located at $\left(\theta_{1}, \theta_{2}\right)=\left( \pm \sqrt{\mu_{\mathrm{r}}} \theta_{\mathrm{E}}, 0\right)$. The magnification of the individual images is computed as

$$
\mu=-\frac{\mu_{\mathrm{r}}^{2}}{2},
$$

which is much smaller than the macro magnification $\mu=\mu_{\mathrm{t}} \mu_{\mathrm{r}}$, indicating that any sources near the origin are significantly less magnified compared with the case without the point mass lens.

\section{Light curves}

In order to predict light curves we need to assume velocities of the lens and source with respect to the observer. The transverse velocity in the lens plane $\mathbf{v}_{l}$ is converted to the angular unit as

$$
\mathbf{u}_{l}=\frac{\mathbf{v}_{l}}{1+z_{l}} \frac{1}{D_{o l}}
$$

where $D_{o l}$ is the angular diameter distance from the observer to the lens and the factor $1+z_{l}$ accounts for the dilution of the unit time; i.e., the angular velocity above indicates the change of the position on the sky per unit observed-frame time. We divide the lens velocity into two components: one is a bulk velocity of the whole lens system (e.g., a peculiar velocity of a galaxy cluster for the case of MACS J1149 LS1), and the other is a relative motion of a point mass lens within the whole lens system. We denote these velocity components as $\mathbf{v}_{m}$ and $\mathbf{v}_{p}$, respectively. We consider these two components separately as they have different dependences on the macro lens model when they are converted to velocities in the source plane (see below).

We also consider the transverse velocity in the source plane $\mathbf{v}_{s}$. Again, it can be converted to the angular unit as

$$
\mathbf{u}_{s}=\frac{\mathbf{v}_{s}}{1+z_{s}} \frac{1}{D_{o s}} .
$$

We derive the relative velocity of the source and lens in the source plane by converting the transverse velocity in the lens plane to the source plane as (see [14])

$$
\mathbf{u}=\mathbf{u}_{m}+\left(\begin{array}{cc}
\mu_{\mathrm{r}}^{-1} & 0 \\
0 & \mu_{\mathrm{t}}^{-1}
\end{array}\right) \mathbf{u}_{p}+\mathbf{u}_{s} .
$$

This indicates that the relative velocity can be anisotropic. The magnification tensor comes in because distances between the point mass lenses in the image plane translate into smaller distances in the source plane due to the cluster potential. The bulk velocity of a cluster is typically $\left|\mathbf{v}_{m}\right| \sim$ $500 \mathrm{~km} \mathrm{~s}^{-1}$ (e.g., [37]). Although the relative motion of the point mass lens, which is of the order of velocity dispersions of massive clusters, $\left|\mathbf{v}_{p}\right| \sim 1000 \mathrm{~km} \mathrm{~s}^{-1}$, is larger than the bulk velocity, it is suppressed by the magnification factors as shown in Eq. (23). The contribution from the source motion is expected to be smaller given the larger redshift and distance to the source. Therefore, a simple approximation which we adopt in the following discussions is

$$
\mathbf{u} \approx \mathbf{u}_{m} .
$$

On the other hand, Fig. 1 indicates that the caustics are elongated along the $x$ axis by a factor of $\sqrt{\mu_{\mathrm{t}}} \gg 1$. 
Thus, caustic crossing typically occurs by a source moving along the $y$ axis with the velocity $\sim\left|\mathbf{u}_{m}\right|$.

When a source crosses the caustics vertically along the $y$ axis, crossing occurs two times in total for the positive parity case, and four times for the negative parity case. As discussed above, for the negative parity case, a source is demagnified near the center.

The behavior of the total magnification near the caustic is important for estimating the possible maximum magnification. The total magnification near the caustics is known to behave as $\mu \propto \Delta \beta^{-1 / 2}$, where $\Delta \beta$ is the distance between the source and the caustic in the source plane. From the analytic examination and numerical calculations with GLAFIC, we find that the magnifications near the caustics are crudely approximated as

$$
\mu(\Delta \beta) \approx \mu_{\mathrm{t}} \mu_{\mathrm{r}}\left(\frac{\theta_{\mathrm{E}}}{\sqrt{\mu_{\mathrm{t}}} \Delta \beta}\right)^{1 / 2},
$$

which agrees with numerical results within a factor of $\lesssim 2$. This approximation is reasonable given that the width of the caustic is "shrunk" by a factor of $\sqrt{\mu_{\mathrm{t}}}$ [Eq. (13)].

Equation (25) suggests that the magnification becomes larger as the source approaches the caustic, which is a universal property of lensing near the caustic (see, e.g., $[38,39])$. However, the magnification saturates when the distance to the caustic becomes comparable to the size of the source in the source plane, $\beta_{R}$ (e.g., [25]). From this condition, we can estimate the maximum magnification as

$$
\mu_{\max } \approx \mu_{\mathrm{t}} \mu_{\mathrm{r}}\left(\frac{\theta_{\mathrm{E}}}{\sqrt{\mu_{\mathrm{t}}} \beta_{R}}\right)^{1 / 2} .
$$

\section{EXPECTED PROPERTIES OF THE LENS AND SOURCE}

\section{A. Dependence on the source star}

We discuss how the peak magnification scales with the radius $R$ and luminosity $L$ of a background star. Assuming a blackbody with temperature $T$, they are related as

$$
\frac{L}{L_{\odot}}=\left(\frac{R}{R_{\odot}}\right)^{2}\left(\frac{T}{T_{\odot}}\right)^{4} .
$$

In practice the spectral energy distribution (SED) of a star does not strictly follow the blackbody, but this relation still holds approximately. Using this relation, we find that the observed maximum flux of the star scales as

$$
f_{\max } \propto \mu_{\max } L \propto R^{-1 / 2} L \propto R^{3 / 2} T^{4} .
$$

Therefore, for a given temperature (which can be inferred from the observation of the SED of the star), the maximum magnified flux of a larger star is larger than that of a smaller star.
If the source size is too big compared with the Einstein radius, we do not observe any microlensing magnifications. From Eq. (26), we can argue that the source size needs to satisfy the following condition to have sensitivity to microlensing:

$$
\sqrt{\mu_{\mathrm{t}}} \beta_{R} \lesssim \theta_{\mathrm{E}}
$$

Suppose that we observe a specific microlensing event in which we can estimate the lower limit of the relative magnification factor during the caustic crossing event, $\mu_{\mathrm{obs}}$, which is derived from the difference of the magnitudes measured at the beginning of the event and when the lensed source is brightest. This gives the lower limit because the true magnification factor during the caustic crossing event is larger given the limited sampling and detection limit of monitoring observations. Thus $\mu_{\mathrm{obs}}$ must be smaller than the relative maximum magnification due to caustic crossing, i.e., $\mu_{\mathrm{obs}}<\mu_{\max } /\left(\mu_{\mathrm{t}} \mu_{\mathrm{r}}\right)$. Using this condition, we can replace the condition in Eq. (29) with

$$
\sqrt{\mu_{\mathrm{t}}} \beta_{R} \lesssim \frac{\theta_{\mathrm{E}}}{\mu_{\mathrm{obs}}^{2}}
$$

This means that, while the peak brightness is higher for the larger star simply because of its large intrinsic brightness, the microlensing magnification is more prominent for the smaller star.

\section{B. Macro model magnification}

The analysis presented in Sec. II assumed a uniform macro model magnification for simplicity. In practice, however, the macro model magnification $\mu_{\mathrm{t}}$ and $\mu_{\mathrm{r}}$ depend on the image position with respect to the critical curve of the macro model. It has been known that $\mu_{\mathrm{t}}$ quickly increases as the image approaches the critical curve (see, e.g., $[38,39])$. More specifically, given that we denote the distance between the image and the critical curve as $\theta_{\mathrm{h}}$, we generally expect $\mu_{\mathrm{t}} \propto \theta_{\mathrm{h}}^{-1}$. We parametrize the dependence on $\theta_{\mathrm{h}}$ as

$$
\mu_{\mathrm{t}}\left(\theta_{\mathrm{h}}\right)=\mu_{\mathrm{h}}\left(\frac{\theta_{\mathrm{h}}}{\operatorname{arcsec}}\right)^{-1},
$$

where $\mu_{\mathrm{h}}$ is a constant factor that depends on the macro lens model, as well as the location on the critical curve. On the other hand, $\mu_{\mathrm{r}}$ is approximately constant near the critical curve.

There is also a well-known asymptotic behavior between $\beta_{\mathrm{h}}$ (the angular distance to the caustic in the source plane) and $\theta_{\mathrm{h}}$ (the angular distance to the critical curve in the image plane), $\beta_{\mathrm{h}} \propto \theta_{\mathrm{h}}^{2}$. In particular, we parametrize it as 


$$
\beta_{\mathrm{h}}=\beta_{0}\left(\frac{\theta_{\mathrm{h}}}{\operatorname{arcsec}}\right)^{2}
$$

From these equations we have $\mu_{\mathrm{t}}=\mu_{\mathrm{h}}\left(\beta_{\mathrm{h}} / \beta_{0}\right)^{-1 / 2}$ for the macro model magnification of one of the merging pairs of images.

The maximum magnification [Eq. (26)] of caustic crossing is larger for larger $\mu_{\mathrm{t}}$, which suggests that stars that are closer to the critical curve can have higher magnifications. However, the authors of [26] (see also [27]) argued that even a small fraction of point mass lenses significantly changes the asymptotic behavior of the macro model magnification toward the critical curve. This is because the Einstein radius of the point mass lens depends on $\mu_{\mathrm{t}}$ as $\propto \sqrt{\mu_{\mathrm{t}}}$, and hence for very large $\sqrt{\mu_{\mathrm{t}}}$ the Einstein radii for different point mass lenses overlap, even when the number density of point mass lenses is small. As shown by the raytracing simulations in [26], beyond this "saturation" point the source breaks into many microimages, and as a result it loses its sensitivity to the source position with respect to the macro model caustic. Therefore, the macro model magnification in fact does not diverge as predicted by Eq. (31), but saturates at a finite value.

To estimate where the saturation happens, the authors of [26] considered the optical depth $\tau$ defined by

$$
\tau=\frac{\Sigma}{M} \pi\left(\sqrt{\mu_{\mathrm{t}}} \theta_{\mathrm{E}} D_{o l}\right)^{2},
$$

where $\Sigma$ is the surface mass density of the point mass component. Here we implicitly assumed that the point masses have the same mass $M$, although we note that this approximation is reasonably good when compared with the realistic ray-tracing simulations. The authors of [26] argued that the saturation happens when $\tau \approx 1$. From the definition of the Einstein radius [Eq. (3)], it is found that

$$
\tau \propto \mu_{\mathrm{t}} \Sigma
$$

which indicates that the maximum macro model magnification where the saturation happens is inversely proportional to the surface mass density of the point mass component $\Sigma$, and it does not depend on the mass $M$. This means that, in order to achieve high peak magnifications [Eq. (26)], lower $\Sigma$ is preferred. Specifically, we can compute the maximum macro model magnification by setting $\tau=1$ in Eq. (33) as

$$
\mu_{\mathrm{t}, \max } \approx \frac{M}{\pi \Sigma\left(\theta_{\mathrm{E}} D_{o l}\right)^{2}} .
$$

Again, for a fixed surface density $\Sigma, \mu_{\mathrm{t}, \max }$ does not depend on mass $M$.

We can consider another condition for the saturation from the Einstein radius (see [27]). Even for $\tau \ll 1$, when the distance to the macro model critical line, $\theta_{\mathrm{h}}$, becomes comparable to the Einstein radius of the point mass lens, the critical curves by the point mass lens merge with those from the macro lens model, and our basic assumption breaks down. Therefore, to have enough magnifications by the point mass lens, we need the following condition:

$$
\sqrt{\mu_{\mathrm{t}}} \theta_{\mathrm{E}} \lesssim \theta_{\mathrm{h}}
$$

Using Eq. (31), this condition is rewritten as

$$
\theta_{\mathrm{E}} \lesssim \frac{\mu_{\mathrm{h}}}{\mu_{\mathrm{t}}^{3 / 2}}
$$

The similar condition in the source plane is

$$
\frac{\theta_{\mathrm{E}}}{\sqrt{\mu_{\mathrm{t}}}} \lesssim \beta_{\mathrm{h}}
$$

which results in

$$
\theta_{\mathrm{E}} \lesssim \frac{\beta_{0} \mu_{\mathrm{h}}^{2}}{\mu_{\mathrm{t}}^{3 / 2}}
$$

In practice, Eqs. (37) and (39) give quite similar conditions, so in what follows we consider only Eq. (37). From this condition, we have another condition for the maximum magnification of the macro mass model as

$$
\mu_{\mathrm{t}, \max } \approx\left(\frac{\mu_{\mathrm{h}}}{\theta_{\mathrm{E}}}\right)^{2 / 3},
$$

which indicates $\mu_{\mathrm{t}, \max } \propto M^{-1 / 3}$. The true maximum macro magnification is given by the smaller of $\mu_{\mathrm{t}, \max }$ given in Eqs. (35) and (40).

\section{Light curve time scales}

There are important time scales that characterize caustic crossing events. One is the so-called source crossing time defined by

$$
t_{\mathrm{src}}=\frac{2 \beta_{R}}{u},
$$

where $\beta_{R}$ is the angular size of the source in the source plane, and $u$ is the source plane velocity as defined in Eq. (23). This source crossing time determines the time scale of the light curve near the peak. Another important time scale is given by the time to cross between caustics. Using the width of the caustics, $\beta_{\mathrm{w}}$ [Eq. (13)], the time to cross between caustics is expressed as

$$
t_{\text {Ein }}=\frac{\beta_{\mathrm{w}}}{u},
$$


which gives the typical time scale between multiple caustic crossing events. The former time scale $t_{\text {src }}$ does not depend on the lens property, whereas the latter time scale $t_{\text {Ein }}$ scales with the lens mass as $\propto M^{1 / 2}$.

\section{Apparent size of microlensed image}

If the Einstein radius is sufficiently large, multiple images can be resolved. In this case, from the separations of the multiple images we can directly infer the mass scale of the lens. In usual cases, however, the Einstein radius of the point mass lens is sufficiently small compared with the angular resolution of observations. As a result, the observed microlensed image is pointlike, from which we can set the constraint on the lens mass as

$$
\sqrt{\mu_{\mathrm{t}}} \theta_{\mathrm{E}} \lesssim \sigma_{\theta, \mathrm{obs}}
$$

where $\sigma_{\theta, \text { obs }}$ is the angular resolution of observations. For the case of Hubble Space Telescope imaging observations, we typically have $\sigma_{\theta, \text { obs }} \approx 0.05$ arcsec.

\section{CONSTRAINING THE SOURCE AND LENS PROPERTIES OF MACS J1149 LS1}

We now tune the parameters to the observations of MACS J1149 LS1 [1] and see what kind of constraints we can place on properties of both the lens object and the source star.

\section{A. Parameters}

The lens redshift of MACS J1149 LS1 is $z_{l}=0.544$ and the source redshift is $z_{s}=1.49$. Both the redshifts are spectroscopic redshifts and therefore are sufficiently accurate. For a cosmology with matter density $\Omega_{M}=0.3$, cosmological constant $\Omega_{\Lambda}=0.7$, and the dimensionless Hubble constant $h=0.7$, we have $6.4 \mathrm{kpc} \mathrm{arcsec}^{-1}$ at the lens and $8.5 \mathrm{kpc} \operatorname{arcsec}^{-1}$ at the source. The distance modulus to the source is 45.2. With these distances, the Einstein radius of the (isolated) point mass lens is

$$
\theta_{\mathrm{E}} \approx 1.8 \times 10^{-6}\left(\frac{M}{M_{\odot}}\right)^{1 / 2} \operatorname{arcsec} .
$$

The angular size of a star as a function of solar radius in the source plane is

$$
\beta_{R} \approx 2.7 \times 10^{-12}\left(\frac{R}{R_{\odot}}\right) \operatorname{arcsec} .
$$

We note that uncertainties of our analysis originating from cosmological parameter uncertainties are much smaller compared with other uncertainties that we will discuss below.

As discussed in Sec. IIC, we can assume that the velocity is dominated by that of the bulk motion of the lensing cluster, $|v| \approx\left|v_{m}\right| \sim 500 \mathrm{~km} \mathrm{~s}^{-1}$. We can convert it to the angular velocity on the sky as

$$
u \approx 5.2 \times 10^{-8}\left(\frac{v}{500 \mathrm{~km} \mathrm{~s}^{-1}}\right) \operatorname{arcsec} \mathrm{yr}^{-1} .
$$

While we fix the $v=500 \mathrm{~km} \mathrm{~s}^{-1}$ in our main analysis, we also check how the uncertainty on the velocity propagates into various constraints that we obtain in this paper.

For the macro mass model using the best-fitting model of the GLAFIC mass model $[36,40]$, we have $\mu_{\mathrm{h}} \approx 13$ in Eq. (31) and $\beta_{0} \approx 0.045$ in Eq. (32). MACS J1149 LS1 was discovered at $\theta_{\mathrm{h}} \approx 0.13 \mathrm{arcsec}$, at which the model predicts the magnification of $\mu_{\mathrm{t}} \approx 100$. On the other hand, $\mu_{\mathrm{r}} \approx 3$ near MACS J1149 LS1. Therefore, the total macro mass model magnification at the position of MACS J1149 LS1 is $\mu_{\mathrm{t}} \mu_{\mathrm{r}} \approx 300$.

We note that there is uncertainty associated with the macro mass model. For example, the authors of [26] noted that the GLAFIC and WSLAP+ [41] mass models of MACS $\mathrm{J} 1149.6+2223$ predict roughly a factor of 2 different macro model magnifications near MACS J1149 LS1 (see also [42] for a test of the accuracy of strong lens mass modeling). This difference in the macro mass model affects our quantitative results. Again, while we fix mass model parameter values to those mentioned above in our analysis, we also examine the dependence of our results on macro mass model uncertainties by checking the dependence of our results on $\mu_{\mathrm{h}}$ that differs considerably between GLAFIC and WSLAP+ mass models.

The ICL plays a crucial role in the interpretation of the caustic crossing event. At the position of MACS J1149 LS1, the surface density of ICL is estimated as $\Sigma_{\mathrm{ICL}} \approx$ 11-19 $M_{\odot} \mathrm{pc}^{-2}$ depending on assumed stellar initial mass functions [1,43]. Given the critical surface density $\Sigma_{\text {crit }} \approx 2.4 \times 10^{3} M_{\odot} \mathrm{pc}^{-2}$, the convergence from the ICL reads $\kappa_{\text {ICL }} \approx 0.0046-0.0079$. This should be compared with the total surface density $\kappa \approx 0.83$ predicted by the bestfitting GLAFIC mass model $[36,40]$. In what follows, we may use the mass fraction of the point mass lens component defined by

$$
f_{\mathrm{p}}=\frac{\Sigma}{2000 M_{\odot} \mathrm{pc}^{-2}},
$$

instead of the surface mass density $\Sigma$.

\section{B. Constraints on MACS J1149 LS1}

Based on discussions given in Sec. III, we constrain the properties of the lens that is responsible for the observed caustic crossing event, as well as the properties of the source star.

First, from Eqs. (26), (44), and (45), at the position of MACS J1149 LS1 with $\mu_{\mathrm{t}} \approx 100$ and $\mu_{\mathrm{r}} \approx 3$, the maximum magnification becomes 


$$
\mu_{\max } \approx 7.8 \times 10^{4}\left(\frac{M}{M_{\odot}}\right)^{1 / 4}\left(\frac{R}{R_{\odot}}\right)^{-1 / 2} .
$$

Assuming the temperature to be $T=12000 \mathrm{~K}$ (see [1]), the absolute $V$-band magnitude of the star before the magnification is computed using Eq. (27) as

$$
M_{\mathrm{star}} \approx 2.2-5 \log \left(\frac{R}{R_{\odot}}\right)
$$

where we used $M_{L_{\odot}} \approx 4.8$ in the $V$-band, $T_{\odot}=5777 \mathrm{~K}$, and included the bolometric correction. Therefore, taking into account the cross-filter K-correction derived in [1], the minimum apparent magnitude of the star magnified by microlensing at the peak is

$$
\begin{aligned}
m_{\text {peak }} & \approx 46.4-5 \log \left(\frac{R}{R_{\odot}}\right)-2.5 \log \mu_{\text {max }}, \\
& \approx 34.1-3.75 \log \left(\frac{R}{R_{\odot}}\right)-0.625 \log \left(\frac{M}{M_{\odot}}\right) .
\end{aligned}
$$

The observation indicates that the peak magnitude is brighter than $m=26$ [1], i.e.,

$$
3.75 \log \left(\frac{R}{R_{\odot}}\right)+0.625 \log \left(\frac{M}{M_{\odot}}\right) \gtrsim 8.1 .
$$

During the caustic crossing event, the source star is magnified by at least a factor of 3 or so. Setting $\mu_{\text {obs }} \approx 3$ in Eq. (30), we obtain a constraint on the source size as

$$
\left(\frac{R}{R_{\odot}}\right)\left(\frac{M}{M_{\odot}}\right)^{-1 / 2} \lesssim 7600
$$

We now consider the condition that the saturation does not happen, because the quantitative constraints derived above assumed no saturation at the position of MACS J1149 LS1. By setting $\mu_{\mathrm{t}}=100$ in Eq. (35), we obtain

$$
\Sigma \lesssim 24 M_{\odot} \mathrm{pc}^{-2}
$$

Again, we caution that this is the result assuming that point mass lenses have the same mass $M$. As shown above, the ICL component has the surface density of $\Sigma_{\text {ICL }} \approx$ 11-19 $M_{\odot} \mathrm{pc}^{-2}$ (which corresponds to the mass fraction of $\left.f_{\mathrm{ICL}}=\Sigma_{\mathrm{ICL}} / \Sigma_{\mathrm{tot}} \approx 0.0055-0.0095\right)$ and therefore satisfies this condition. On the other hand, from the other condition for nonsaturation [Eq. (40)], we have

$$
M \lesssim 5.1 \times 10^{7} M_{\odot} .
$$

For MACS J1149 LS1, the source crossing time [Eq. (41)] is

$$
t_{\mathrm{src}} \approx 0.038\left(\frac{R}{R_{\odot}}\right)\left(\frac{v}{500 \mathrm{~km} \mathrm{~s}^{-1}}\right)^{-1} \text { days. }
$$

Similarly, the time scale between caustic crossings is

$$
t_{\text {Ein }} \approx 3.5\left(\frac{M}{M_{\odot}}\right)^{1 / 2}\left(\frac{v}{500 \mathrm{~km} \mathrm{~s}^{-1}}\right)^{-1} \mathrm{yr} .
$$

In the case of MACS J1149 LS1, the source crossing time, which is the time scale where the light curve is affected by the finiteness of the source star radius very near the peak, appears to be smaller than $\sim 10$ days [1]. The condition $t_{\text {src }} \lesssim 10$ days becomes

$$
\left(\frac{R}{R_{\odot}}\right)\left(\frac{v}{500 \mathrm{~km} \mathrm{~s}^{-1}}\right)^{-1} \lesssim 260 .
$$

For this source size, the apparent magnitude of the star without microlensing by the point mass lens is $\lesssim 28$, which appears to be consistent with the observation [1]. Also $t_{\text {Ein }}$ seems to be at least larger than $\sim 1 \mathrm{yr}$, so $t_{\text {Ein }} \gtrsim 1 \mathrm{yr}$ gives rise to

$$
\left(\frac{M}{M_{\odot}}\right)\left(\frac{v}{500 \mathrm{~km} \mathrm{~s}^{-1}}\right)^{-2} \gtrsim 0.082 .
$$

Since MACS J1149 LS1 was unresolved during the caustic crossing event, we use Eq. (43) to set the constraint on the mass of the lens as

$$
M \lesssim 7.6 \times 10^{6} M_{\odot} .
$$

By using this argument we may also exclude the possibility that the caustic crossing event was produced by massive dark matter substructures.

We now put together all these constraints and derive allowed ranges of the lens mass $M$ and the source size $R$. The result is shown in Fig. 2, where we fixed the bulk velocity of the lens to $v=500 \mathrm{~km} \mathrm{~s}^{-1}$. We find that there are large ranges of the lens mass and the source size that can explain MACS J1149 LS1.

However, it is expected that the lens and source populations are not distributed uniformly in this parameter space. As discussed in the next section, the size distribution of the source star is expected to be significantly bottom heavy; i.e., stars with smaller radii are more abundant than those with larger radii. The same argument also holds for the lens mass, if we assume standard stars and stellar remnants as the lens population, but with the minimum mass of $\approx 0.3 M_{\odot}$ below which the stellar initial mass function is truncated. Therefore, in the allowed parameter space, the most likely set of parameters is $R \approx 180 R_{\odot}$ and $M \approx 0.3 M_{\odot}$. In this case, the star is magnified by a factor of $\approx 4300$ at the peak. The result is fully consistent with the 


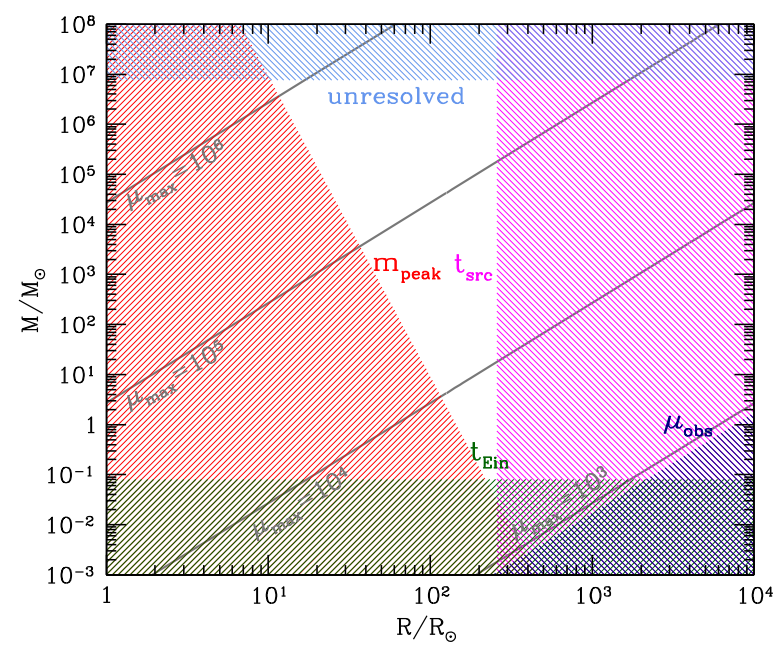

FIG. 2. Constraints on the source radius $(R)$ and lens mass $(M)$ for MACS J1149 LS1. Shaded regions show excluded regions from various constraints. Specifically, we consider constraints from the peak magnitude $\left[m_{\text {peak }}\right.$, Eq. (51)]; the magnification during the caustic crossing $\left[\mu_{\mathrm{obs}}\right.$, Eq. (52)]; the source crossing time $\left[t_{\text {src }}\right.$, Eq. (57)]; the time scale between caustic crossings $\left[t_{\text {Ein }}\right.$, Eq. (58)], and the unresolved shape during caustic crossing [unresolved, Eq. (59)]. The saturation condition given by Eq. (54) is always satisfied in the allowed region of this plot. Contours show the constant peak magnification [Eq. (48)] in this parameter space. From top to bottom, we show contours of $\mu_{\max }=10^{6}, 10^{5}$, $10^{4}$, and $10^{3}$.

scenario that a blue supergiant is magnified by a foreground ICL star.

On the other hand, our result does not exclude the possibility that the microlensing is caused by an exotic population such as PBHs with masses between $\sim 1 M_{\odot}$ and $\sim 10^{6} M_{\odot}$, as long as they have low surface density so that they satisfy the saturation condition. For more massive lenses, Fig. 2 indicates that the peak magnification is even higher and can reach up to $\sim 10^{4}-10^{6}$.

\section{EVENT RATES}

\section{A. Star population in the arc}

It is estimated that the surface brightness of the arc is $\approx 25 \mathrm{mag} \mathrm{arcsec}^{-2}$ in the F125W band [1], which corresponds to $\approx 6.5 \times 10^{9} L_{\odot} \operatorname{arcsec}^{-2}$. We need to convert the observed arc surface brightness to the number density of stars that can be magnified by caustic crossing events. We do so by simply assuming a power-law luminosity function of stars, $d n / d L \propto L^{-2}$, as considered in [1]. The normalization of the luminosity function is determined so that the total luminosity density $\int_{L_{\min }}^{L_{\max }} L(d n / d L) d L$ matches the observed surface brightness. Assuming the luminosity range of $L_{\min }=0.1 L_{\odot}$ and $L_{\max }=10^{7} L_{\odot}$, the surface number density can be converted to the number density of stars in the image plane

$$
\begin{aligned}
n_{\text {star }}\left(L_{1}<L<L_{2}\right) & =\frac{6.5 \times 10^{9} \operatorname{arcsec}^{-2}}{\mu_{\mathrm{t}} \mu_{\mathrm{r}} \ln \left(L_{\max } / L_{\min }\right)}\left(\frac{L_{\odot}}{L_{1}}-\frac{L_{\odot}}{L_{2}}\right) \\
& \approx \frac{3.5 \times 10^{8} \operatorname{arcsec}^{-2}}{\mu_{\mathrm{t}} \mu_{\mathrm{r}}}\left(\frac{L_{\odot}}{L_{1}}-\frac{L_{\odot}}{L_{2}}\right),
\end{aligned}
$$

where $\mu_{\mathrm{t}} \mu_{\mathrm{r}}$ in the denominator accounts for the lensing magnification of luminosities of individual stars. Using Eq. (27) and fixing $T=12000 \mathrm{~K}$, we can convert this to the number density in the star radius range

$$
n_{\text {star }}\left(R_{1}<R<R_{2}\right)=\frac{n_{0}}{\mu_{\mathrm{t}} \mu_{\mathrm{r}}}\left[\left(\frac{R_{\odot}}{R_{1}}\right)^{2}-\left(\frac{R_{\odot}}{R_{2}}\right)^{2}\right],
$$

where $n_{0}=1.9 \times 10^{7} \operatorname{arcsec}^{-2}$. For a given lens mass $M$, the lower limit of the radius comes from the constraint on the peak magnitude. Here we consider caustic crossing events with $m_{\text {peak }}<26$. From Eq. (50)

$$
R_{1} \approx 140\left(\frac{\mu_{\mathrm{t}}}{100}\right)^{-0.5}\left(\frac{M}{M_{\odot}}\right)^{-0.167} R_{\odot}
$$

where we recover the dependence on $\mu_{\mathrm{t}}$ which originates from Eq. (26). We set $R_{2} \approx 730 R_{\odot}$, the radius corresponding to $L_{\max }=10^{7} L_{\odot}$.

\section{B. Expected rate}

From the analysis in Sec. II, we know that the typical length scale of the caustic along the $\beta_{1}$ direction is $\approx 2\left(\sqrt{\mu_{\mathrm{t}}} / \mu_{\mathrm{r}}\right) \theta_{\mathrm{E}}$. Therefore the expected event rate is

$$
\begin{aligned}
\frac{d N}{d t}= & 2 \int_{\theta_{\mathrm{h}, \min }}^{\theta_{\mathrm{h}, \max }} d \theta_{\mathrm{h}} n_{\mathrm{star}} w_{\mathrm{arc}} \mu_{\mathrm{t}} \mu_{\mathrm{r}} \frac{\Sigma}{M} 2 \frac{\sqrt{\mu_{\mathrm{t}}}}{\mu_{\mathrm{r}}} D_{o l}^{2} \theta_{\mathrm{E}} u, \\
& +\left.2 \int_{0}^{\theta_{\mathrm{h}, \min }} d \theta_{\mathrm{h}} n_{\mathrm{star}} w_{\mathrm{arc}} \mu_{\mathrm{t}} \mu_{\mathrm{r}} \frac{\Sigma}{M} 2 \frac{\sqrt{\mu_{\mathrm{t}}}}{\mu_{\mathrm{r}}} D_{o l}^{2} \theta_{\mathrm{E}} u\right|_{\mu_{\mathrm{t}, \max }} \\
= & 2 \int_{\mu_{\mathrm{t}, \text { min }}}^{\mu_{\mathrm{t}, \max }} \frac{\mu_{\mathrm{h}} d \mu_{\mathrm{t}}}{\mu_{\mathrm{t}}^{2}} n_{0}\left[\left(\frac{R_{\odot}}{R_{1}}\right)^{2}-\left(\frac{R_{\odot}}{R_{2}}\right)^{2}\right] \\
& \times w_{\mathrm{arc}} \frac{\Sigma}{M} 2 \frac{\sqrt{\mu_{\mathrm{t}}}}{\mu_{\mathrm{r}}} D_{o l}^{2} \theta_{\mathrm{E}} \\
& +\left.2 \theta_{\mathrm{h}, \text { min }} n_{\mathrm{star}} w_{\mathrm{arc}} \mu_{\mathrm{t}} \mu_{\mathrm{r}} \frac{\Sigma}{M} 2 \frac{\sqrt{\mu_{\mathrm{t}}}}{\mu_{\mathrm{r}}} D_{o l}^{2} \theta_{\mathrm{E}} u\right|_{\mu_{\mathrm{t}, \max }},
\end{aligned}
$$

where $w_{\text {arc }}$ (assumed to be 0.2 arcsec in the following calculations) is the width of the giant arc along the critical curve, and the saturation conditions give the upper limit $\mu_{\mathrm{t}, \max }$. The factor $\mu_{\mathrm{t}} \mu_{\mathrm{r}}$ converts the number density of the point mass lens in the image plane, $\Sigma / M$, to the corresponding number density in the source plane. The prefactor 2 is introduced due to the fact that caustic crossing events can happen on both sides of the critical curve. As shown in Sec. II, while the length of the caustic is shorter in the negative parity region, there are twice as many caustic 
crossings for each lens, which would compensate for the shorter length of the caustic. The second term represents the contribution from the saturated region in which caustic crossing events are observed (see [26,27]). We make a simple assumption that the rate calculation of the saturated region is the same as that for the unsaturated region but with replacing $\mu_{\mathrm{t}}$ to the saturation value $\mu_{\mathrm{t}, \max }$. Among the saturation conditions given in Eqs. (35) and (40), parameter values of $\Sigma$ [or equivalently $f_{\mathrm{p}}$, defined in Eq. (47)] and $M$ determine which condition determines the maximum $\mu_{\mathrm{t}}$. These two conditions reduce to

$$
\begin{gathered}
\mu_{\mathrm{t}, \max }=1.2 f_{\mathrm{p}}^{-1}, \\
\mu_{\mathrm{t}, \max }=3.7 \times 10^{4}\left(\frac{M}{M_{\odot}}\right)^{-1 / 3} .
\end{gathered}
$$

By equating these two conditions, we can define the critical point mass fraction $f_{\mathrm{p} \text {,crit }}$ :

$$
f_{\text {p,crit }}=3.2 \times 10^{-5}\left(\frac{M}{M_{\odot}}\right)^{1 / 3} .
$$

When $f_{\mathrm{p}}>f_{\mathrm{p}, \text { crit }}, \mu_{\mathrm{t}, \max }$ is determined from Eq. (64), whereas when $f_{\mathrm{p}}<f_{\mathrm{p} \text {,crit }}, \mu_{\mathrm{t}, \max }$ is determined from Eq. (65). One condition to determine $\mu_{\mathrm{t}, \text { min }}$ is $R_{1}=R_{2}$, which gives $\mu_{\mathrm{t}, \min } \lesssim 1$. In practice, $\mu_{\mathrm{t}, \min }$ would be determined by the extent of the arc in the direction perpendicular to the critical curve. We tentatively set $\mu_{\mathrm{t}, \min }=10$, which corresponds to the maximum distance from the critical curve, $\theta_{\mathrm{h}, \max } \approx 1.3^{\prime \prime}$ [see Eq. (31)]. In some cases, however, $\mu_{\mathrm{t}, \text { min }}$ determined from $R_{1}=R_{2}$ becomes larger than 10, and in that case we adopt the former value as $\mu_{\mathrm{t} \text { min }}$.

Plugging in the parameter values for MACS J1149 LS1, we have

$$
\begin{aligned}
& \frac{d N}{d t} \approx 5.0 \times 10^{5} f_{\mathrm{p}}\left(\frac{M}{M_{\odot}}\right)^{-1 / 2}\left(\frac{v}{500 \mathrm{~km} \mathrm{~s}^{-1}}\right) I(M) \\
& +4.5 \times 10^{4} f_{\mathrm{p}}\left(\frac{M}{M_{\odot}}\right)^{-1 / 2}\left(\frac{v}{500 \mathrm{~km} \mathrm{~s}^{-1}}\right) J(M), \\
& I(M)=\int_{\mu_{\mathrm{t}, \text { min }}}^{\mu_{\mathrm{t}, \max }} \frac{d \mu_{\mathrm{t}}}{\mu_{\mathrm{t}}^{3 / 2}}\left[\left(\frac{R_{\odot}}{R_{1}}\right)^{2}-\left(\frac{R_{\odot}}{R_{2}}\right)^{2}\right] \\
& \approx 9.3 \times 10^{-7}\left(\mu_{\mathrm{t}, \max }^{1 / 2}-\mu_{\mathrm{t}, \min }^{1 / 2}\right)\left(\frac{M}{M_{\odot}}\right)^{1 / 3} \\
& +3.7 \times 10^{-6}\left(\mu_{\mathrm{t}, \max }^{-1 / 2}-\mu_{\mathrm{t}, \min }^{-1 / 2}\right), \\
& J(M)=\left(\frac{R_{\odot}}{\left.R_{1}\right|_{\mu_{\mathrm{t}, \max }}}\right)^{2}-\left(\frac{R_{\odot}}{R_{2}}\right)^{2} \\
& \approx 4.7 \times 10^{-7} \mu_{\mathrm{t}, \max }\left(\frac{M}{M_{\odot}}\right)^{1 / 3}-1.9 \times 10^{-6} \text {, }
\end{aligned}
$$

where $d N / d t$ is the event rate, i.e., the number of caustic crossing events per year.

We compute the event rate as a function of lens mass $M$ and mass fraction of the lens $f_{\mathrm{p}}$ using Eq. (67). We can use this predicted rate calculation to place additional constraints on the lens population. Since MACS J1149 LS1 is observed with $\sim 2$ years of monitoring observations of MACS J1149, the $2 \sigma$ limit of the predicted rate is

$$
\frac{d N}{d t} \gtrsim 0.025 \text { year }^{-1}
$$

Here we do not consider the additional event (Iapyx) in the rate constraint because its peak brightness may be fainter than 26 mag.

Figure 3 shows the constraint from the event rate [Eq. (70)] in the $M-f_{\mathrm{p}}$ plane. We find that there exists an allowed region with $M \lesssim 4 \times 10^{3} M_{\odot}$ and $f_{\mathrm{p}} \lesssim 0.3$. For a fixed $f_{\mathrm{p}}$, large lens masses result in low event rates because the mean free path of a source is proportional to $M^{-1 / 2}$, whereas small lens masses also result in low event rates because of the lower maximum magnification [see Eq. (48)]. Interestingly, this allowed region is fully consistent with the ICL component, which has the right mass range and mass fraction. Therefore, together with the result shown in Fig. 2, we conclude that the observation of MACS J1149 LS1 is fully explained by microlensing due to an ICL star.

When marginalized over $f_{\mathrm{p}}$, the result in Fig. 3 provides an additional constraint on the mass range of the point lens

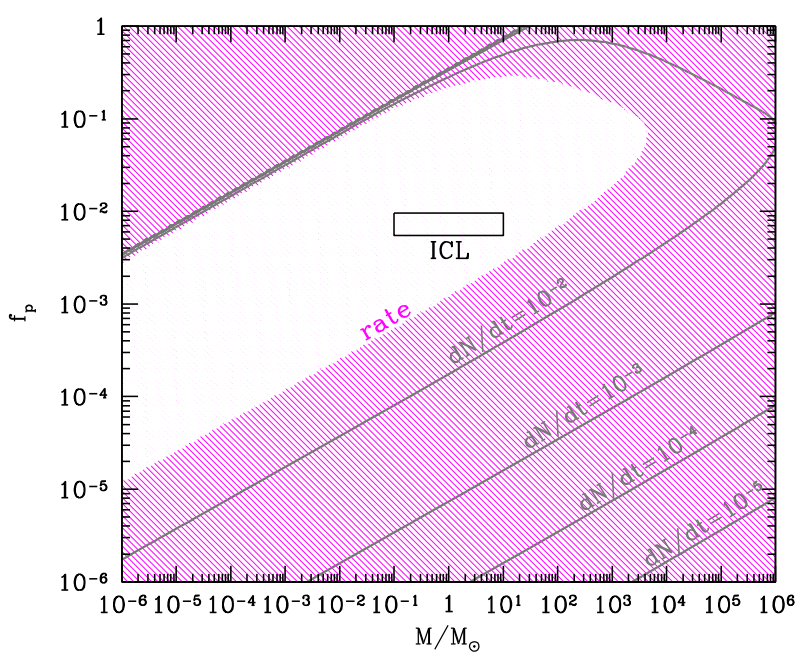

FIG. 3. Constraints in the $M-f_{\mathrm{p}}$ plane for MACS J1149 LS1, where $M$ is the lens mass and $f_{\mathrm{p}}$ is the mass fraction of the point mass component to the total mass. Shaded regions show excluded regions from the event rate [rate, Eq. (70)]. The small rectangular region shows the rough mass fraction and the mass range of ICL stars. Contours show the constant event rate in this plane. From inner to outer contours, we show contours for $d N / d t=10^{-2}$, $10^{-3}, 10^{-4}$, and $10^{-5}$. 


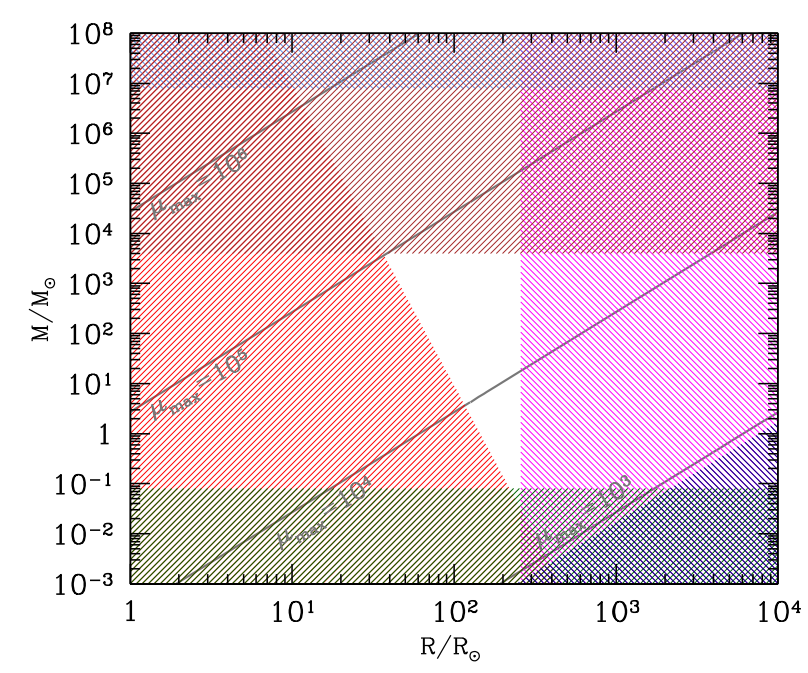

FIG. 4. Similar to Fig. 2, but an additional constraint on the lens mass range from the event rate (see Fig. 3) is included.

component that produced MACS J1149 LS1. Thus, in Fig. 4, we revisit the constraints in the $R-M$ plane, with the additional constraint from Fig. 3. We find that the additional constraint makes the allowed ranges of the lens mass and source size narrower. However, the most plausible values of $R \approx 180 R_{\odot}$ and $M \approx 0.3 M_{\odot}$ assuming the prior distributions (see discussions in Sec. IV B) are kept unchanged by this additional constraint. This new constraint from the event rate limits more severely the possibility of explaining MACS J1149 LS1 by exotic dark matter models such as PBHs.

Are there any ways to further constrain the lens mass? One possible way is to check the positions of caustic crossing events. Because point mass lenses with larger masses can produce higher magnifications due to caustic crossing, the macro lens magnification $\mu_{\mathrm{t}}$ required to exceed the peak magnitude threshold can be smaller. This means that microlensing by large mass lenses can be observed at positions farther away from the critical curve of the macro mass model. We check this point explicitly by computing distributions of the distance from the macro model critical curve, $\theta_{\mathrm{h}}$, for different lens masses. The result shown in Fig. 5, which plots the normalized differential distribution of the event rate as a function of $\theta_{\mathrm{h}}$, indicates that this is indeed the case. With just one event it is impossible to conclude which lens mass is favored, but by observing many caustic crossing events near the critical curve, we may be able to constrain the mass of the point mass lens component more directly.

Another way to better constrain the lens mass is to observe multiple caustic crossing events by a single lens, because the time interval between those events provides information on the mass of the lens [see Eq. (42)]. For instance, the light curve of MACS J1149 LS1 shows a possible peak in the spring of 2014 [1]. If this is interpreted as a caustic crossing event from the same lens, the

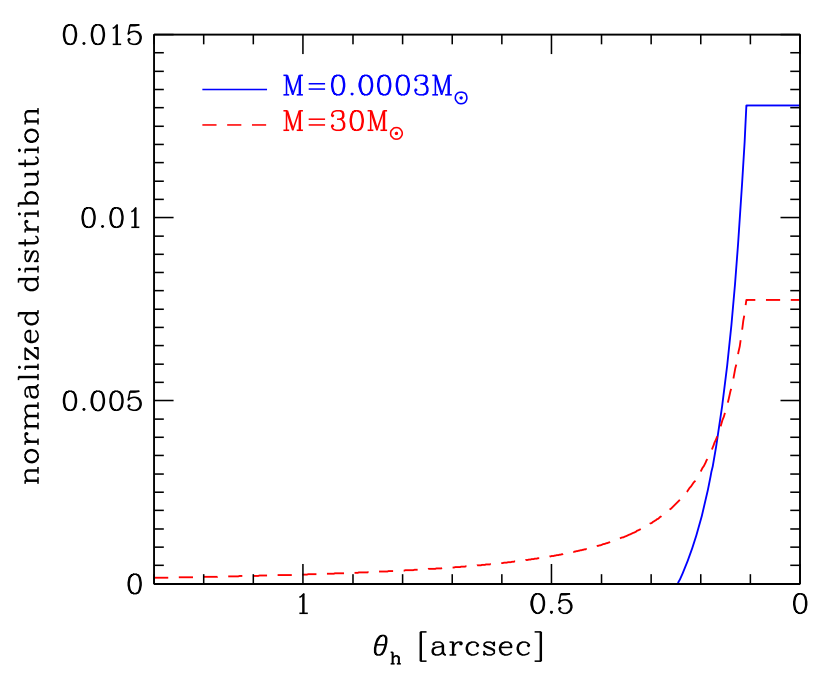

FIG. 5. The expected distribution of the positions of caustic crossing events from the macro model critical curve $\theta_{\mathrm{h}}$, i.e., the normalized differential distribution of the event rate, $\left(d^{2} N / d \theta_{\mathrm{h}} d t\right) /\left(\int d \theta_{\mathrm{h}} d^{2} N / d \theta_{\mathrm{h}} d t\right)$. The distribution is essentially the integrand of Eq. (63). Parameters are tuned for those of MACS J1149 LS1 as considered in Sec. IV B. We consider two different masses of the point mass lens component, $M=$ $0.0003 M_{\odot}$ (solid) and $30 M_{\odot}$ (dashed). Given the relation given in Eq. (31), the distribution of $\theta_{\mathrm{h}}$ can also be converted to that of the macro model magnification $\mu_{\mathrm{t}}$.

observation suggests $t_{\text {Ein }} \approx 2$ years, which implies $M \approx$ $0.3 M_{\odot}$ from Eq. (56), which is fully consistent with other constraints derived in this paper.

\section{Effects of model parameter uncertainties}

Predictions of event rates and constraints on lens and source properties described in the previous subsection are subject to model parameter uncertainties. Among others, the assumption on the transverse velocity is a source of large uncertainties given that the probability distribution of the velocity is quite broad, with the width of the distribution being a factor of 2 [37]. In addition, as discussed in Sec. IV, the macro model magnification may also involve a large uncertainty, which can have a large impact on our results. Here we explore effects of model parameter uncertainties on our results, focusing on uncertainties associated with the transverse velocity $v$ and the macro model magnification $\mu_{\mathrm{h}}$ defined in Eq. (31), assuming the uncertainty of \pm 0.5 dex (i.e., factors of 2 and 0.5 ) on these parameters.

First, we discuss uncertainties of predicted event rates due to uncertainties of $v$ and $\mu_{\mathrm{h}}$. From Eq. (63), it is found that the event rate is linearly proportional to both $v$ and $\mu_{\mathrm{h}}$. Therefore, the uncertainty of \pm 0.5 dex on $v$ and $\mu_{\mathrm{h}}$ directly translates into the uncertainty of \pm 0.5 dex on the predicted event rate. This indicates that our prediction on the event rate for Icarus is uncertainty by a factor of 2 or so.

Next, we check the effects of the uncertainty on these parameters on our constraints on lens and source properties 


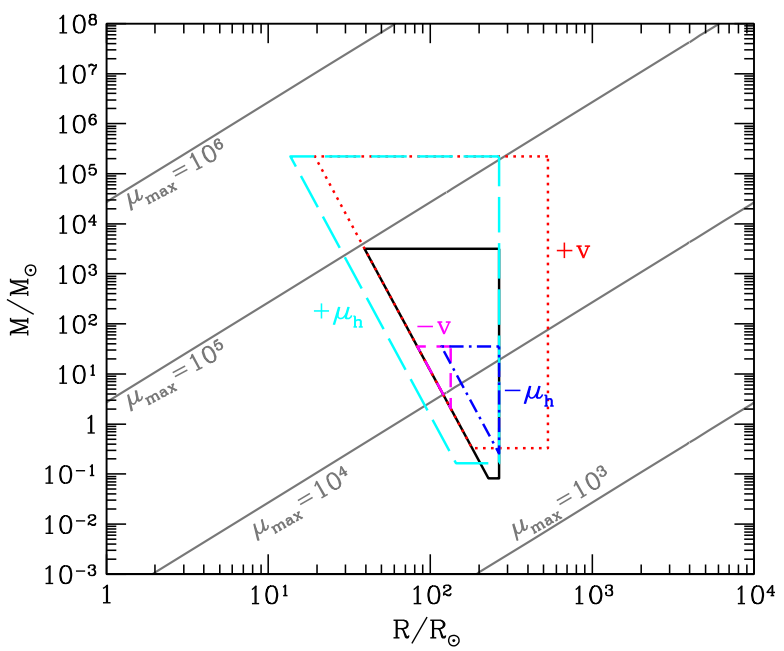

FIG. 6. Dependence of constraints in the $R-M$ plane shown in Fig. 4 on model parameters. The solid line indicates an allowed region in the $R-M$ plane in our fiducial setup, as shown in Fig. 4. Dotted and short dashed lines show how the allowed region changes by changing the transverse velocity $v$ by factors of 2 and 0.5 , respectively. Long dashed and dotted-dashed lines show how the allowed region changes by changing the macro model magnification $\mu_{\mathrm{h}}$ by factors of 2 and 0.5 , respectively.

that are summarized in Fig. 4. In Fig. 6, we show how the allowed region shown in Fig. 4 changes by shifting $v$ and $\mu_{\mathrm{h}}$ by \pm 0.5 dex. We find that the impact of these model parameter uncertainties on our results is indeed significant. Interestingly, the lens mass of $\sim 1 M_{\odot}$ and the source star radius of $\sim 100 R_{\odot}$ is allowed even if we take into account these model parameter uncertainties.

\section{Dependence of event rates on model parameters}

The expected event rate given by Eq. (63) depends on various model parameters. To understand the model dependence on the event rate, we repeat the computation of the event rate for MACS J1149 LS1, changing one of the model parameters while fixing the other model parameters. Here we change the source redshift $z_{s}$, the surface brightness of the arc, and the limiting magnitude of the monitoring observation $m_{\text {lim }}$. We show the result in Fig. 7 .

We find that the event rate is relatively a steep function of the source redshift $z_{s}$. This is simply because we need higher magnification in order for stars at higher redshifts to be observed. Since the observed maximum flux is an increasing function of the source radius $R$ [see Eq. (28)], stars detected in giant arcs at higher redshifts correspond to intrinsically more luminous stars. The event rate becomes zero beyond $z_{s} \sim 3.8$, because there is no star that has the observed maximum flux that exceeds the detection limit $m_{\text {limm }}$.

The dependence on the surface brightness is easily understood. The number of stars is proportional to the total luminosity of the arc, and increasing the surface brightness with a fixed arc size simply increases the total luminosity.
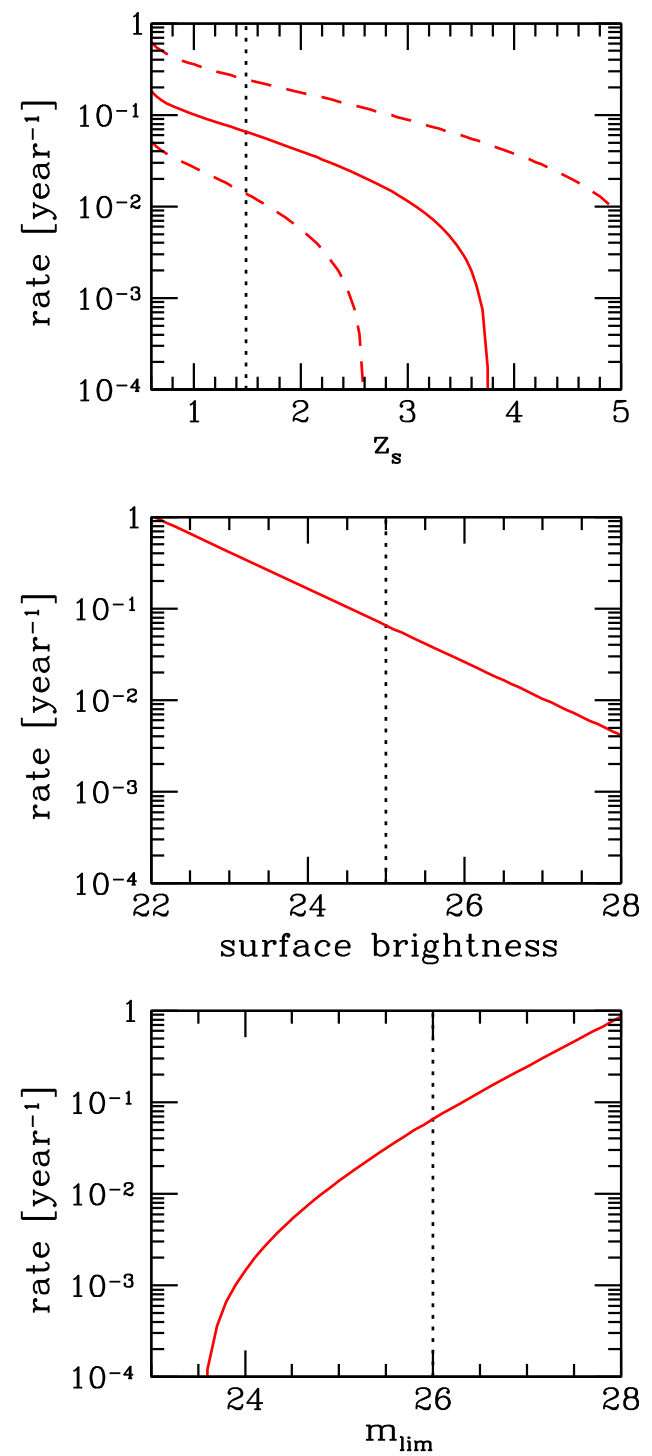

FIG. 7. The dependences of the event rate [Eq. (63)] on various model parameters. We consider model parameters that are tuned for the MACS J1149 LS1 (see Sec. IV B), and fix the mass fraction of the point mass lens $f_{\mathrm{p}}=0.01$ and the lens mass $M=0.3 M_{\odot}$. From the top panel to the bottom, we show the dependences of the event rate on the source redshift $z_{s}$, the surface brightness of the arc, and the limiting magnitude of the monitoring observation $m_{\text {lim }}$. The vertical dotted lines show our fiducial values for MACS J1149 LS1. In the top panel, we also show the results for the limiting magnitudes brighter and fainter by $1 \mathrm{mag}$ (i.e., $m_{\mathrm{lim}}=25$ and 27 ) by dashed lines.

We also find that the dependence of the event rate on the limiting magnitude $m_{\mathrm{lim}}$ is strong. For example, by monitoring with 2 magnitude deeper images, which can be enabled with the James Webb Space Telescope, we may be able to detect $\sim 10$ times more caustic crossing events, allowing more-detailed statistical studies of the caustic crossing events such as the spatial distribution, as discussed in Sec. VB. Again, Eq. (28) indicates that deeper observations allow us to detect less luminous stars, which are more abundant. 
There is a sharp cutoff at $m_{\lim } \sim 23.5$ for the same reason as in the source redshift $z_{s}$.

\section{CONSTRAINTS ON COMPACT DARK MATTER IN THE PRESENCE OF ICL}

As discussed in [1,26,27], even though ICL stars can fully explain MACS J1149 LS1, we can still place constraints on the compact dark matter scenario where some fraction of dark matter consists of compact objects such as black holes. This is because such compact dark matter can break the caustic due to the macro lens model into microcaustics, which reduce the magnification significantly (see discussions in Sec. III B). The high fraction of compact dark matter leads to significant saturation at the position of MACS J1149 LS1, which effectively reduces the macro model magnification at that position. Since the smaller macro model magnification leads to fainter peak magnitudes of caustic crossing events, the high level of saturation can be inconsistent with the observation of MACS J1149 LS1.

We can quantify the constraint as follows. From the peak magnitude [Eq. (50)] and the constraint on the source radius [Eq. (57)], we can derive the minimum (brightest) peak magnitude as

$$
\begin{aligned}
m_{\text {peak }, \min } \approx & 25.1-0.625 \log \left(\frac{M}{M_{\odot}}\right) \\
& -1.875 \log \left(\frac{\mu_{\mathrm{t}, \mathrm{LS} 1}}{100}\right),
\end{aligned}
$$

where $\mu_{\mathrm{t}, \mathrm{LS} 1}$ is given by Eq. (64) for the case of interest here. For compact dark matter with masses $M<10 M_{\odot}$, we conservatively assume that the MACS J1149 LS1 is produced by an ICL star with the mass $10 M_{\odot}$ because larger lens masses predict brighter peak magnitudes. Given the condition $m_{\text {peak,min }}<26$ we obtain the following constraint on $f_{\mathrm{p}}$ :

$$
f_{\mathrm{p}}<0.08 \text {. }
$$

For compact dark matter with masses $M>10 M_{\odot}$, we can achieve brighter peak magnitudes by assuming that the caustic crossing event was produced by compact dark matter rather than an ICL star. In this case, from the same condition $m_{\text {peak,min }}<26$, we obtain

$$
\log f_{\mathrm{p}}<-1.44+\frac{1}{3} \log \left(\frac{M}{M_{\odot}}\right)
$$

The condition given in Eq. (72) is in principle independent of the mass of the compact dark matter microlens, which means that this constraint can be applied for a wide mass range below $10 M_{\odot}$. However, when the mass of the compact dark matter microlens is very small, the extent of the caustic produced by compact dark matter becomes

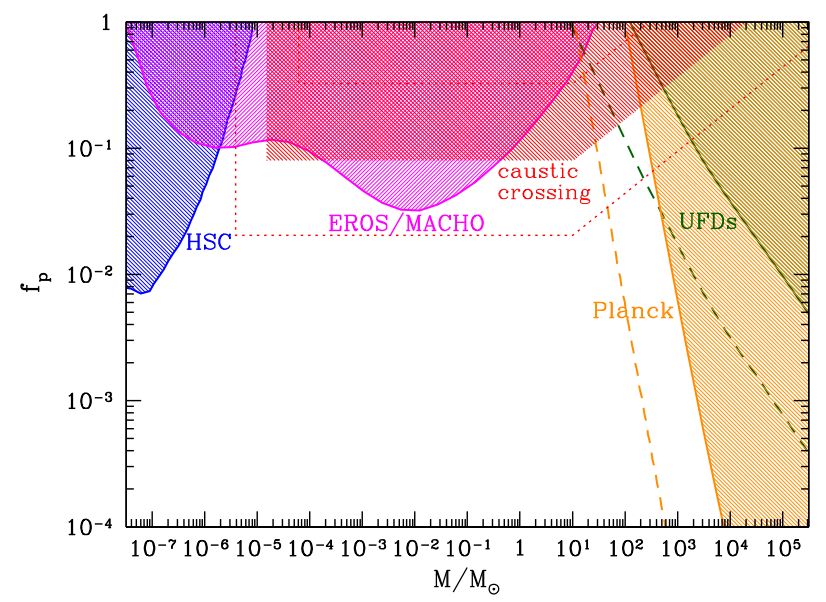

FIG. 8. Constraints on the mass $(M)$ and abundance $\left(f_{\mathrm{p}}\right)$ of compact dark matter. Shaded regions show excluded regions from the caustic crossing studied in this paper, microlensing observations of M31 with Subaru/Hyper Suprime-Cam (HSC) [11], EROS/MACHO microlensing [6,9], ultrafaint dwarf galaxies (UFDs) [45], and Planck cosmic microwave background observations (Planck) [46]. We also show constraints from the caustic crossing with different assumptions on the transverse velocity (factors of 2 and 0.5 different from the fiducial value) by dotted lines. For UFDs and Planck, conservative limits are shown by solid lines, whereas more stringent limits are shown by dashed lines. For the Planck constraint, the stringent limit assumes the collisional ionization around $\mathrm{PBHs}$, whereas the conservative limit assumes the photoionization due to the $\mathrm{PBH}$ radiation. For the UFDs constraint, different constraints reflect different assumptions on the dark matter densities and initial sizes of star clusters in UFDs.

much smaller than the source size. In this case, any lensing effects by compact dark matter are smeared out due to the finite source size effect, and as a result it does not cause any saturation. We can write this condition as

$$
\frac{\theta_{\mathrm{E}}}{\sqrt{\mu_{\mathrm{t}}}} \lesssim \beta_{R}
$$

Given the allowed range of the source radius $R$ and $\mu_{\mathrm{t}}<100$, this condition reduces to

$$
M \lesssim 1.5 \times 10^{-5} M_{\odot}
$$

From this argument, we can derive constraints on the mass $M$ and abundance $f_{\mathrm{p}}$ of compact dark matter. Figure 8 shows the rough excluded region in the $M-f_{\mathrm{p}}$ plane from the observation of MACS J1149 LS1. As discussed in [1], the very high abundance of $\sim 30 M_{\odot}$ black holes [28], which is motivated by recent observations of gravitational waves [44], is excluded, although more careful comparisons with simulated microlensing light curves should be made in order to place more robust constraints.

As discussed in Sec. V C, our constraints are subject to model parameter uncertainties. While we find that the 
uncertainty of the macro model magnification $\mu_{\mathrm{h}}$ does not affect our constraint on $f_{\mathrm{p}}$ from the saturation condition, the uncertainty on the transverse velocity $v$ is expected to have a large impact on our result via the dependence of the maximum source star radius on the velocity [Eq. (57)]. To evaluate this, we repeat the analysis presented above with different values of the transverse velocity by \pm 0.5 dex, i.e., factors of 2 and 0.5 different from the original value of $v=500 \mathrm{~km} \mathrm{~s}^{-1}$. The resulting constraints shown in Fig. 8 indicate that our results on the compact dark matter abundance are indeed sensitive to the assumed transverse velocity. We find that the constraint on $f_{\mathrm{p}}$ is weaker for the higher velocity, because Eq. (57) suggests that larger source radii (i.e., intrinsically brighter source stars) are allowed for the higher velocity. We find that the very high abundance of $\sim 30 M_{\odot}$ black holes are still excluded even for the high velocity case, which is encouraging. In order to draw more robust constraints on $f_{\mathrm{p}}$, we need to convolve our constraints on the probability distributions of the transverse velocity, as well as other model parameters, which we leave for future work.

We expect that we can place tighter constraints on compact dark matter from long monitoring observations of giant arcs and careful analysis of observed light curves. This is because point mass lenses with different masses have quite different characteristics of light curves such as time scales and peak magnifications. Therefore, observations or absence of light curve peaks with different time scales may be used to place constraints on the abundance of compact dark matter with different masses, although we have to take into account the uncertainty in the velocity for the robust interpretation. As discussed in [26], another clue may be obtained by detailed observations of light curves before and after the peak. As mentioned above, in order to obtain robust constraints on compact dark matter from observations, it is also important to conduct ray-tracing simulations that include both ICL stars and compact dark matter, as was partly done in [26]. Ray-tracing simulations are helpful to better understand what kind of light curves such compound lens systems predict.

\section{SUMMARY AND DISCUSSIONS}

In this paper, we have adopted a simple analytical lens model that consists of a point mass lens and a constant convergence and shear field, which is used to study lensing properties of a point mass lens embedded in high magnification regions due to the cluster potential. This model has been used to derive characteristic scales of caustic crossing events in giant arcs, such as the time scale of light curves and maximum magnifications, as a function of the mass of the point mass lens and the radius of the source star. We have tuned model parameters to the MACS J1149 LS1 event to constrain lens and source properties of this event. We have also computed expected event rates, and derived additional constraints on the lens and source properties of MACS J1149 LS1.

Our results that are summarized in Figs. 3 and 4 indicate that MACS J1149 LS1 is fully consistent with microlensing by ICL stars. The allowed ranges of the lens mass and source radius are $0.1 M_{\odot} \lesssim M \lesssim 4 \times 10^{3} M_{\odot}$ and $40 R_{\odot} \lesssim R \lesssim 260 R_{\odot}$, respectively. The most plausible radius of the source star is $R \approx 180 R_{\odot}$ (luminosity $L \approx 6 \times 10^{5} L_{\odot}$ ), which is consistent with a blue supergiant. In this case, the source star should have been magnified by a factor of $\approx 4300$ at the peak. Our results suggest that the allowed ranges of the lens mass and source radius are relatively narrow, which limits the possibility of explaining MACS J1149 LS1 with exotic dark matter models.

We have discussed the possibility of constraining compact dark matter in the presence of ICL stars. Using the saturation argument, we have shown that compact dark matter models with high fractional matter densities $\left(f_{\mathrm{p}} \gtrsim 0.1\right)$ for a wide mass range of $10^{-5} M_{\odot} \lesssim$ $M \lesssim 10^{2} M_{\odot}$ are inconsistent with the observation of MACS J1149 LS1 because such models predict too low magnifications at the position of MACS J1149 LS1. We note that this constraint from the saturation condition should be applicable to the total compact dark matter fraction for models with extended mass functions [47]. We expect that we can place tighter constraints on the abundance and mass of compact dark matter by careful analysis of observed light curves as well as more observations of caustic crossing events.

In this paper, we have assumed a single star as a source. As discussed in [1], there is a possibility that the source is in fact a binary star, based on multiple peaks in the light curve. Even for a binary star system, our results are broadly applicable to individual stars that constitute the binary system.

There are several additional caveats in our analysis. As discussed in the paper, our constraints sensitively depend on the assumption of the velocity $v$ as well as the macro model magnification. To draw a more robust conclusion, we have to take into account the distributions of the velocity and the macro model magnification. We can also consider more realistic star models, such as an improved mass-radius relation of stars beyond the blackbody relation [Eq. (27)] and a more realistic population of stars with various temperatures.

We also did not discuss the "counterimage" (Iapyx) of MACS J1149 LS1 presented in [1]. The position of the second image, which was separated by 0.26 " from MACS J1149 LS1, is consistent with being the counterimage. The authors of [1] argued that a point mass lens with $M \gtrsim 3 M_{\odot}$ is needed to demagnify the counterimage for several years. From Eq. (11), we can estimate the time scale of the demagnification as $t_{\mathrm{demag}} \approx 2 \beta_{\text {crit, }+} / u \approx 14\left(M / M_{\odot}\right)^{1 / 2}$ year, which suggests that indeed a point mass lens with $M \sim 3 M_{\odot}$ 
is capable of demagnifying the counterimage for many years. In order for this conclusion to hold, the counterimage must be bright enough to be detected in the absence of microlensing. From Eq. (50), we can estimate the apparent brightness of the counterimage without microlensing magnification (but with the macro model magnification) to be $\approx 28.9 \mathrm{mag}$ for $R=180 R_{\odot}$, which is much fainter than the limiting magnitude of the monitoring observation. However, the source radius is allowed to be as large as $R=260 R_{\odot}$ (see Fig. 4), which suggests that the counterimage can be as bright as $\approx 28.1$ mag without microlensing, which can marginally be detected in individual observations of MACS J1149. In observations, while a source with the magnitude $\approx 28$ was observed in previous images [1], given the expected fluctuations of light curves and the limited time coverage of observations, it is not clear whether this really corresponds to the brightness of the source for the macro model magnification $\mu_{\mathrm{t}} \mu_{\mathrm{r}}=300$. Therefore, the conclusion about the demagnification of the counterimage crucially depends on the intrinsic radius (luminosity) of the source star. There is also a possibility that this is in fact not a counterimage but a distinct star magnified by microlensing. While this may be more plausible given the relatively low probability of caustic crossing events for individual source stars, simulations in [1] indicate that a single star tends to be responsible for the vast majority of the detectable microlensing peaks. This, together with the rarity of blue supergiant stars, prefers the scenario that Icarus and Iapyx originate from the same star.

An additional caveat is that substructures can also change the relative macro model magnifications of
Icarus and Iapyx, as noted in [1]. While the standard cold dark matter naturally predicts such dark halo substructures, compact dark matter with relatively large masses can produce more fluctuations on the macro model magnification due to the Poisson fluctuations of the projected surface mass density as a function of position on the sky. Such spatial variation of the macro model magnifications should have an impact on our quantitative results, including constraints on compact dark matter from the saturation argument (Sec. VI). We leave the exploration of this effect to future work.

To summarize, our analytic examinations have demonstrated that observations of caustic crossing events in giant arcs have a great potential to study the nature of dark matter. Our predictions on characteristic scales and event rates should provide useful guidance for future monitoring of giant arcs in clusters for obtaining various constraints from caustic crossing events.

\section{ACKNOWLEDGMENTS}

We thank an anonymous referee for useful suggestions. This work was supported in part by World Premier International Research Center Initiative (WPI Initiative), MEXT, Japan, and JSPS KAKENHI Grants No. JP26800093 and No. JP15H05892. J. M. D. acknowledges the support of Projects No. AYA2015-64508-P (MINECO/FEDER, UE), No. AYA2012-39475-C02-01, and the consolider Project No. CSD2010-00064 funded by the Ministerio de Economia y Competitividad.
[1] P. L. Kelly et al., arXiv:1706.10279.

[2] P. L. Kelly et al., Science 347, 1123 (2015).

[3] S. A. Rodney et al., arXiv:1707.02434.

[4] C. Alcock et al. (Supernova Cosmology Project Collaboration), Nature (London) 365, 621 (1993).

[5] A. Udalski et al., Acta Astronomica 44, 165 (1994).

[6] C. Alcock et al. (MACHO Collaboration), Astrophys. J. 542, 281 (2000).

[7] T. Sumi et al., Astrophys. J. 591, 204 (2003).

[8] J. T. A. de Jong et al. (MEGA Collaboration), Astron. Astrophys. 446, 855 (2006).

[9] P. Tisserand et al. (EROS-2 Collaboration), Astron. Astrophys. 469, 387 (2007).

[10] K. Griest, A. M. Cieplak, and M. J. Lehner, Phys. Rev. Lett. 111, 181302 (2013).

[11] H. Niikura et al., arXiv:1701.02151.

[12] K. Chang and S. Refsdal, Nature (London) 282, 561 (1979).

[13] B. Paczynski, Astrophys. J. 301, 503 (1986).

[14] R. Kayser, S. Refsdal, and R. Stabell, Astron. Astrophys. 166, 36 (1986).
[15] H. J. Witt, Astron. Astrophys. 236, 311 (1990).

[16] P. L. Schechter and J. Wambsganss, Astrophys. J. 580, 685 (2002).

[17] J. Wambsganss, B. Paczynski, and P. Schneider, Astrophys. J. 358, L33 (1990).

[18] R. Schmidt and J. Wambsganss, Astron. Astrophys. 335, 379 (1998).

[19] D. Pooley, J. A. Blackburne, S. Rappaport, and P. L. Schechter, Astrophys. J. 661, 19 (2007).

[20] G. Chartas, C. S. Kochanek, X. Dai, S. Poindexter, and G. Garmire, Astrophys. J. 693, 174 (2009).

[21] C. W. Morgan, C. S. Kochanek, N. D. Morgan, and E. E. Falco, Astrophys. J. 712, 1129 (2010).

[22] J. A. Blackburne, D. Pooley, S. Rappaport, and P. L. Schechter, Astrophys. J. 729, 34 (2011).

[23] J. Jiménez-Vicente, E. Mediavilla, C. S. Kochanek, and J. A. Muñoz, Astrophys. J. 806, 251 (2015).

[24] E. Mediavilla, J. Jiménez-Vicente, J. A. Muñoz, H. VivesArias, and J. Calderón-Infante, Astrophys. J. 836, L18 (2017). 
[25] J. Miralda-Escudé, Astrophys. J. 379, 94 (1991).

[26] J. M. Diego et al., arXiv:1706.10281.

[27] T. Venumadhav, L. Dai, and J. Miralda-Escud, Astrophys. J. 850, 49 (2017).

[28] S. Bird, I. Cholis, J. B. Muñoz, Y. Ali-Haïmoud, M. Kamionkowski, E. D. Kovetz, A. Raccanelli, and A. G. Riess, Phys. Rev. Lett. 116, 201301 (2016).

[29] M. Sasaki, T. Suyama, T. Tanaka, and S. Yokoyama, Phys. Rev. Lett. 117, 061101 (2016).

[30] M. Kawasaki, A. Kusenko, Y. Tada, and T. T. Yanagida, Phys. Rev. D 94, 083523 (2016).

[31] B. Carr, F. Kuhnel, and M. Sandstad, Phys. Rev. D 94, 083504 (2016).

[32] K. Inomata, M. Kawasaki, K. Mukaida, Y. Tada, and T. T. Yanagida, Phys. Rev. D 96, 043504 (2017).

[33] W. Hu, R. Barkana, and A. Gruzinov, Phys. Rev. Lett. 85, 1158 (2000).

[34] H. Y. Schive, T. Chiueh, and T. Broadhurst, Nat. Phys. 10, 496 (2014).

[35] L. Hui, J. P. Ostriker, S. Tremaine, and E. Witten, Phys. Rev. D 95, 043541 (2017).

[36] M. Oguri, Publ. Astron. Soc. Jpn. 62, 1017 (2010).
[37] K. Dolag and R. Sunyaev, Mon. Not. R. Astron. Soc. 432, 1600 (2013).

[38] R. Blandford and R. Narayan, Astrophys. J. 310, 568 (1986).

[39] P. Schneider, J. Ehlers, and E. E. Falco, Gravitational Lenses (Springer, New York, 1992).

[40] R. Kawamata, M. Oguri, M. Ishigaki, K. Shimasaku, and M. Ouchi, Astrophys. J. 819, 114 (2016).

[41] J. M. Diego, T. Broadhurst, C. Chen, J. Lim, A. Zitrin, B. Chan, D. Coe, H. C. Ford, D. Lam, and W. Zheng, Mon. Not. R. Astron. Soc. 456, 356 (2016).

[42] M. Meneghetti et al., Mon. Not. R. Astron. Soc. 472, 3177 (2017).

[43] T. Morishita, L. E. Abramson, T. Treu, K. B. Schmidt, B. Vulcani, and X. Wang, Astrophys. J. 846, 139 (2017).

[44] B. P. Abbott et al. (LIGO Scientific and Virgo Collaborations), Phys. Rev. Lett. 116, 061102 (2016).

[45] T. D. Brandt, Astrophys. J. 824, L31 (2016).

[46] Y. Ali-Haïmoud and M. Kamionkowski, Phys. Rev. D 95, 043534 (2017).

[47] B. Carr, M. Raidal, T. Tenkanen, V. Vaskonen, and H. Veerme, Phys. Rev. D 96, 023514 (2017). 